\title{
Estimating completeness of cancer registration: an empirical evaluation of the two source capture-recapture approach in Germany
}

\author{
Hermann Brenner, Christa Stegmaier, Hartwig Ziegler
}

\begin{abstract}
Study objective - In recent years, capturerecapture methods have become increasingly popular in estimating completeness of disease registries. This study aimed to assess the performance of the two source capture-recapture method in estimating the completeness of cancer registration.

Design - The study was conducted in the population based cancer registry of Saarland, Germany, for which there are three main sources of notifications: reports by clinicians and pathologists, and death certificates. For groups of cases notified by one of the three sources, known completeness of registration by the other two sources was compared with the corresponding two source capture-recapture estimates.
\end{abstract}

Patients - A total of 16020 patients notified to the cancer registry in $1970,1975,1980$, and 1985 were included in the analysis.

Main results - There was a tendency towards underestimation of completeness of notifications from pathologists and death certificates for patients notified by clinicians which was essentially confined to the older age groups. In contrast, capturerecapture methods tended to overestimate completeness of notifications from clinicians and death certificates for patients notified by pathologists. This overestimation was observed consistently in all age groups and for all of the most common cancer sites. Nevertheless, deviations of estimated completeness from known completeness were generally small or moderate. Agreement between estimated and known completeness was closest for patients notified by death certificates, although completeness was somewhat underestimated in patients above age 75 . The observed patterns are in agreement with knowledge on clinical aspects and clinical management of cancer patients and with the circumstances of cancer registration in Saarland.

Conclusions - Careful application of capture-recapture methods may provide an alternative to traditional approaches for estimating completeness of cancer registration.

( $\mathcal{F}$ Epidemiol Community Health 1995;49:426-430)
Population based cancer registries are important tools of cancer epidemiology in that they provide the basis for monitoring cancer incidence over time and between populations or between population subgroups. Furthermore, they often assist in case identification in analytical epidemiological studies. A prerequisite for these tasks is a high level of completeness of case registration. ${ }^{1}$ Unfortunately, there seems to be no standard tool to assess the completeness of cancer registration. The most commonly used indices of completeness - the death certificate only index (DCO index) and the mortality/incidence ratio (M/I ratio) ${ }^{2}$ are indirect indices which are strongly superimposed by the case fatality rate. Furthermore, they have been used inconsistently by various disease registries. $^{3}$

Recently, capture-recapture methods have gained increasing popularity as a way of estimating directly the completeness of disease registries. ${ }^{4-9}$ Capture-recapture methodology was originally developed and has been widely used in wildlife science as a tool for estimating the size of free-living animal populations. ${ }^{10}$ Briefly, samples of animals are captured, tagged, released, and recaptured, and the size of the animal population is estimated from the numbers of animals captured and recaptured in each sample. The analogy with disease registration is to consider cases registered by different sources as separate samples from the population of all cases, and to derive estimates of the total size of this latter population accordingly. ${ }^{11}$ In the context of cancer registration, such sources might include notifications by clinicians, radiologists, pathologists, or notifications through death certificates.

A well known major limitation of capturerecapture methods is their sensitivity to their underlying assumptions. In the simplest situation, in which only two sources of case identification are available, the assumption of independence between these two sources has to be made. ${ }^{12-14}$ In the context of disease registration, this assumption may often be violated. ${ }^{14}$ For example, cases of disorders with a lengthy duration or more severe cases are often more likely to be reported by multiple sources than other cases. This results in a positive dependence of ascertainment rates and in underestimation of the total number of cases (or overestimation of completeness of registration, accordingly). In other situations, the sources may be mutually exclusive, leading to 
a negative dependence and overestimation of the total number of cases (or underestimation of completeness, accordingly). Evaluation of the independence assumption and the sequelae of their potential violation requires comparison of cases registered by both sources with an external, independent list of cases. With very few exceptions, ${ }^{15}$ such empirical evaluation is lacking. In this paper, we provide an empirical assessment of the performance of the two source capture-recapture approach in estimating the completeness of cancer registration using data from the population based cancer registry of Saarland/Germany.

\section{Methods}

The cancer registry of Saarland, established in 1966, registers incident cases of cancer among the approximately 1.05 million inhabitants of Saarland. It is the only population based cancer registry in the western part of Germany that is generally considered to have provided reasonably accurate and complete incidence data throughout the past two decades. ${ }^{316}$ Our analysis is based on all cases of malignant neoplasm (ICD-9; 140-208) excluding non-melanoma skin cancer (ICD-9; 173) notified to the cancer registry in 1970, 1975, 1980, and 1985 . There are multiple sources of notification of cancer cases. The most important among these are clinicians (including hospital doctors and physicians in private practice) and pathologists. In addition, death certificates of residents of Saarland are routinely linked with the cancer registry which provides an additional important source of notification. If cases are first notified by a death certificate, the cancer registry of Saarland routinely requests an additional notification from the clinician who filled out the death certificate in order to obtain more complete information about these patients.

To assess the performance of the two sample capture-recapture method, the known number of cases notified by one of the three major sources (clinicians, pathologists, and death certificates) was compared with the number estimated from the intersections of notifications from the two other sources among these cases. This is illustrated in table 1 for cases reported by pathologists.

For these cases, the quantities $\mathrm{n}_{11}, \mathrm{n}_{10}, \mathrm{n}_{01}$, and $\mathrm{n}_{00}$ and their sum $\mathrm{n}_{++}$are all known. In the absence of notifications from pathologists, however, only the quantities $n_{11}, n_{10}$, and $n_{01}$ would be known, and the number of cases missed by both death certificates and by clinicians' reports would have to be estimated. Assuming independence of ascertainment by clinicians and death certificates, the maximum likelihood estimate (MLE) for this quantity is

Table 1 Distribution of patients reported by pathologists in relation to the presence or absence of notifications from clinicians and death certificates

\begin{tabular}{lll}
\hline Death certificates & \multicolumn{2}{l}{ Clinicians } \\
\cline { 2 - 3 } & Yes & No \\
\hline Yes & $\mathrm{n}_{11}$ & $\mathrm{n}_{10}$ \\
No & $\mathrm{n}_{01}$ & $\mathrm{n}_{00}$ \\
\hline
\end{tabular}

given as $\hat{n}_{00}=n_{10} n_{01} / n_{11}$, and the MLE for $n_{++}$ is given as $\hat{n}_{++}=\left(n_{10}+n_{11}\right) \quad\left(n_{01}+n_{11}\right) / n_{11}{ }^{13}$ Completeness of notification by clinicians and death certificates is known to be $\left(n_{11}+n_{10}+n_{01}\right) / n_{++}$, but would be estimated to be $\left(n_{11}+n_{10}+n_{01}\right) / n_{++}$in the absence of notifications from pathologists.

We compared estimated and known completeness to assess the performance of the two sample capture-recapture approach. It should be noted that completeness defined in this way does not reflect overall completeness of the cancer registry. Rather, it reflects the proportion of patients among cases notified by pathologists who are also reported by clinicians or death certificates. Analogous analyses are provided for the group of patients notified by clinicians and for the group of patients notified by death certificates which are characterised according to the presence or absence of notifications by the two complementary sources. The analyses were stratified by calendar year of diagnosis, age, and cancer site to allow for identification of eventual differences in performance of the two sample capture-recapture approach between subgroups of patients defined by these variables.

\section{Results}

The overall numbers of cancer patients notified by the three main sources individually and jointly are given in the figure. Each single source captured most of the patients. While most patients were reported by more than one source, each of the three sources contributed a substantial number of patients that would not otherwise have been reported. For example, $8 \%$ of the patients were reported by death certificate only.

Table 2 shows known (K) and estimated (E) completeness of ascertainment by the two complementary sources for patients notified by clinicians, pathologists, and death certificates, stratified by calendar year. Both known and estimated completeness were in the range of

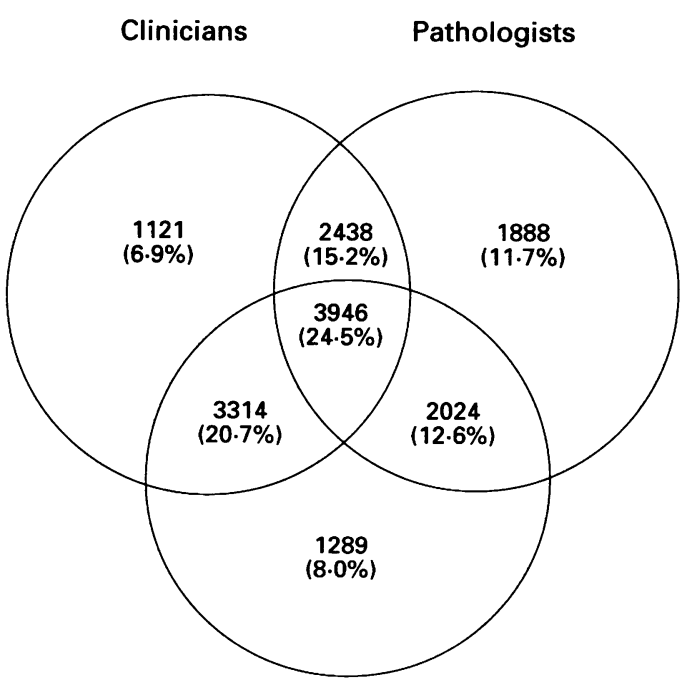

Death certificates

Cancer cases diagnosed in 1970, 1975, 1980, and 1985 , and reported to the cancer registry of Saarland by sources of notification. 
Table 2 Known $(K)$ and estimated (E) completeness of notifications from the two complementary sources in patients notified to the cancer registry of Saarland by clinicians, pathologists, and death certificates. Stratification by calendar year

\begin{tabular}{|c|c|c|c|c|c|c|c|c|c|}
\hline \multirow[t]{2}{*}{ Calendar year } & \multicolumn{3}{|c|}{ Clinicians } & \multicolumn{3}{|c|}{ Pathologists } & \multicolumn{3}{|c|}{ Death certificates } \\
\hline & No & $K^{*}$ & $E$ & No & $K^{*}$ & $E$ & No & $K^{*}$ & $E$ \\
\hline $\begin{array}{l}1970 \\
1975 \\
1980 \\
1985\end{array}$ & $\begin{array}{l}2744 \\
2861 \\
2577 \\
2637\end{array}$ & $\begin{array}{l}87 \cdot 8 \\
89 \cdot 8 \\
90 \cdot 5 \\
90 \cdot 6\end{array}$ & $\begin{array}{l}81 \cdot 6 \\
84 \cdot 3 \\
85 \cdot 5 \\
81 \cdot 4\end{array}$ & $\begin{array}{l}1682 \\
2596 \\
2775 \\
3243\end{array}$ & $\begin{array}{l}88 \cdot 5 \\
85 \cdot 4 \\
82 \cdot 8 \\
74 \cdot 2\end{array}$ & $\begin{array}{l}92 \cdot 8 \\
89 \cdot 1 \\
86 \cdot 0 \\
81 \cdot 1\end{array}$ & $\begin{array}{l}2582 \\
2635 \\
2737 \\
2619\end{array}$ & $\begin{array}{l}87 \cdot 6 \\
90 \cdot 5 \\
85 \cdot 2 \\
88 \cdot 0\end{array}$ & $\begin{array}{l}86 \cdot 9 \\
87 \cdot 4 \\
85 \cdot 5 \\
82 \cdot 6\end{array}$ \\
\hline
\end{tabular}

* Bold figures indicate significant dependence $(p<0.05)$ between notifications from the two complementary sources.

Table 3 Known $(K)$ and estimated $(E)$ completeness of notifications from the two complementary sources in patients notified to the cancer registry of Saarland by clinicians, pathologists, and death certificates. Stratification by age

\begin{tabular}{|c|c|c|c|c|c|c|c|c|c|}
\hline \multirow[t]{2}{*}{ Age (y) } & \multicolumn{3}{|c|}{ Clinicians } & \multicolumn{3}{|c|}{ Pathologists } & \multicolumn{3}{|c|}{ Death certificates } \\
\hline & No & $K^{*}$ & $E$ & No & $K^{*}$ & $E$ & No & $K^{*}$ & $E$ \\
\hline $\begin{array}{r}0-14 \\
15-29 \\
30-44 \\
45-59 \\
60-74 \\
\geq 75\end{array}$ & $\begin{array}{r}76 \\
171 \\
736 \\
2556 \\
4882 \\
2397\end{array}$ & $\begin{array}{l}69 \cdot 7 \\
75 \cdot 4 \\
83 \cdot 6 \\
89 \cdot 3 \\
91 \cdot 4 \\
89 \cdot 9\end{array}$ & $\begin{array}{l}70 \cdot 3 \\
79 \cdot 0 \\
86 \cdot 2 \\
87 \cdot 6 \\
84 \cdot 8 \\
76 \cdot 7\end{array}$ & $\begin{array}{r}38 \\
162 \\
763 \\
2643 \\
4755 \\
1935\end{array}$ & $\begin{array}{l}86 \cdot 8 \\
69 \cdot 1 \\
79 \cdot 9 \\
83 \cdot 0 \\
83 \cdot 1 \\
77 \cdot 8\end{array}$ & $\begin{array}{l}89 \cdot 8 \\
89 \cdot 8 \\
86 \cdot 8 \\
89 \cdot 2 \\
88 \cdot 3 \\
83 \cdot 4\end{array}$ & $\begin{array}{r}48 \\
87 \\
460 \\
2088 \\
4928 \\
2962\end{array}$ & $\begin{array}{l}89 \cdot 6 \\
94 \cdot 3 \\
93 \cdot 3 \\
94 \cdot 3 \\
89 \cdot 3 \\
79 \cdot 7\end{array}$ & $\begin{array}{l}87 \cdot 0 \\
93 \cdot 2 \\
93 \cdot 2 \\
91 \cdot 6 \\
85 \cdot 4 \\
69 \cdot 2\end{array}$ \\
\hline
\end{tabular}

* Bold figures indicate significant dependence $(p<0.05)$ between notifications from the two complementary sources.

Table 4 Known $(K)$ and estimated $(E)$ completeness of notifications from the two complementary sources in patients notified to the cancer registry of Saarland by clinicians, pathologists, and death certificates. Stratification by main diagnostic groups

\begin{tabular}{|c|c|c|c|c|c|c|c|c|c|}
\hline \multirow[t]{2}{*}{ Cancer site (ICD-9) } & \multicolumn{3}{|c|}{ Clinicians } & \multicolumn{3}{|c|}{ Pathologists } & \multicolumn{3}{|c|}{ Death certificates } \\
\hline & No & $K^{*}$ & $E$ & No & $K^{*}$ & $E$ & No & $K^{*}$ & $E$ \\
\hline $\begin{array}{l}\text { Gastrointestinal tract } \\
(150-154)\end{array}$ & 2313 & $92 \cdot 4$ & $84 \cdot 3$ & 2690 & $79 \cdot 4$ & $84 \cdot 4$ & 2592 & $88 \cdot 1$ & $81 \cdot 9$ \\
\hline $\begin{array}{l}\text { Trachea, bronchi, lung } \\
\text { pleura }(162,163,165)\end{array}$ & 1772 & $92 \cdot 9$ & $91 \cdot 6$ & 1072 & $94 \cdot 6$ & $97 \cdot 0$ & 1932 & $86 \cdot 9$ & 89.9 \\
\hline $\begin{array}{l}\text { Breast }(174,175) \\
\text { Female genital organs } \\
(179-184)\end{array}$ & $\begin{array}{l}1508 \\
1264\end{array}$ & $\begin{array}{l}90 \cdot 7 \\
86 \cdot 2\end{array}$ & $\begin{array}{l}85 \cdot 8 \\
82 \cdot 7\end{array}$ & $\begin{array}{l}1645 \\
1234\end{array}$ & $\begin{array}{l}81 \cdot 3 \\
82 \cdot 0\end{array}$ & $\begin{array}{l}88 \cdot 0 \\
90 \cdot 0\end{array}$ & $\begin{array}{l}824 \\
662\end{array}$ & $\begin{array}{l}93 \cdot 4 \\
91 \cdot 2\end{array}$ & $\begin{array}{l}95 \cdot 2 \\
95 \cdot 2\end{array}$ \\
\hline $\begin{array}{l}\text { Prostate (185) } \\
\text { Urinary tract (188-189) }\end{array}$ & $\begin{array}{l}470 \\
536\end{array}$ & $\begin{array}{l}86 \cdot 8 \\
88 \cdot 6\end{array}$ & $\begin{array}{l}78 \cdot 4 \\
79 \cdot 5\end{array}$ & $\begin{array}{l}643 \\
734\end{array}$ & $\begin{array}{l}65 \cdot 5 \\
64 \cdot 9\end{array}$ & $\begin{array}{l}76 \cdot 9 \\
79 \cdot 4\end{array}$ & $\begin{array}{l}434 \\
468\end{array}$ & $\begin{array}{l}84 \cdot 6 \\
88 \cdot 0\end{array}$ & $\begin{array}{l}81 \cdot 5 \\
83 \cdot 7\end{array}$ \\
\hline
\end{tabular}

* Bold figures indicate significant dependence $(p<0.05)$ between notifications from the two complementary sources.

$80-90 \%$ in most analyses. For patients notified by clinicians, two sample capture-recapture estimates of completeness of notifications from pathologists and death certificates were consistently lower than known levels of completeness, indicating negative dependence between reports from pathologists and death certificates. In contrast, completeness was consistently overestimated among patients notified by pathologists, which indicates positive dependence between notifications by clinicians and death certificates. In all calendar years, agreement between estimated and true completeness was closest for those patients notified by death certificates.

The agreement between estimated and known completeness among patients notified by death certificate was very close in all age groups below 60 , whereas completeness tended to be underestimated by the two source capture-recapture approach above this age (table 3). A similar age dependency was also observed for the patients notified by clinicians. In these patients, the two source capture-recapture estimates of completeness of notifications from pathologists and death certificates were slightly higher than known completeness at younger ages, while completeness was underestimated in the older age groups. In contrast, there was consistent overestimation of completeness of notifications from clinicians and death cer- tificates among patients notified by pathologists over all age groups. In these patients, overestimation of completeness also persisted after stratification by categories of major cancer sites (table 4), whereas consistent underestimation occurred within categories of cancer site for patients notified by clinicians. With the exception of cancers of the gastrointestinal and respiratory tract, agreement between estimated and known completeness was again highest among cases notified by death certificates for all of the most common types of cancer.

\section{Discussion}

Although the two source capture-recapture estimates of completeness differed from the known values in our analysis, this deviation was generally of small or moderate size. In all cases, the deviation was less severe than the bias which is made by ignoring missed cases altogether (that is, by implicitly assuming $100 \%$ completeness of registration), a practice that widely prevails in reporting of cancer incidence rates.

The crucial assumption for the two source capture-recapture method is that of independence between both sources. ${ }^{13}$ Although it is not possible to check formally this assumption if no other sources of ascertainment are available, one might often be able to judge 
whether this assumption is reasonable under the specific circumstances of registration, and, if not, what would be the likely direction of its violation. If positive dependence is more plausible, the capture-recapture approach should be regarded as providing an estimate of an upper limit of completeness, whereas an estimate of a lower limit is provided in the case of presumed negative dependence.

In our analysis, capture-recapture estimates of completeness were most accurate for notifications from clinicians and pathologists of patients notified by death certificates. This may seem surprising at first sight since one might expect positive dependence between reports from clinicians and pathologists. For example, patients who undergo surgery are more likely to be reported by both surgeons and pathologists than other patients. On the other hand, the practice of the Saarland cancer registry to request additional information from the clinician who filled out the death certificate among patients first notified by death certificates is likely to introduce negative dependence between notifications by clinicians and pathologists, since such additional notifications by clinicians would not be obtained for patients previously notified by pathologists. This explanation was supported by further analyses in which notifications by clinicians made upon request after a first notification by death certificate were eliminated. In these analyses, the capture-recapture approach tended to overestimate completeness of notifications from clinicians and pathologists, indicating positive dependence between these two sources.

Our analyses also provide evidence supporting positive dependence between notifications by clinicians and death certificates, which is reflected in overestimation of completeness of notification by these sources in patients reported by pathologists. This seems plausible since patients who eventually die from their cancer are expected to have more extensive contacts with clinicians than other patients.

On the other hand, there was some underestimation of completeness of notifications by pathologists and death certificates among patients notified by clinicians. The underlying negative dependence between notifications by the former two sources could reflect the fact that pathology reports are less common for advanced cancers with poorer prognosis. For example, cancers that are inoperable at the time of diagnosis are typically not seen by the pathologist.

The reported overall patterns of dependency were very similar in the four calendar years included in this analysis. There was some interesting variation, however, of the observed patterns in relation to age and cancer site. For example, underestimation of completeness of notifications by pathologists and death certificates among patients notified by clinicians was essentially confined to the older age groups (which include most cancer patients) and particularly those over 75 years. This seems plausible, given the fact that cancers are more often detected at an advanced, inoperable stage in this age group. Patients with such cancers have higher case fatality rates, and their cancer is less often seen by a pathologist than other cancers.

Overestimation of completeness of notifications by clinicians and death certificates in patients notified by pathologists was observed consistently in all age groups. The somewhat larger extent of overestimation in the age group 15-29 years should be interpreted with caution due to the relatively small number of patients in this age group.

Although known and estimated completeness of notifications by clinicians and pathologists was generally rather close among patients notified by death certificates, considerable underestimation was observed in patients aged above 75 . This could reflect more frequent contacts with clinicians along with a lower probability of surgery and histological confirmation of tumours in this age group.

Stratification by cancer site revealed the following patterns. Among patients notified by clinicians as well as among patients notified by pathologists, true completeness of notifications by the complementary sources was highest for cancers of the respiratory tract. This is because most of these cancers, which have a very poor prognosis, are notified through death certificates.

Some underestimation of completeness of notification by pathologists and death certificates was observed for all main diagnostic groups among patients notified by clinicians. This underestimation was more pronounced for gastrointestinal cancers and cancers of the prostate and urinary tract than for other cancers. For the same cancers, completeness of notification was also underestimated among patients notified by death certificates. These observations may primarily reflect the age related patterns discussed above since gastrointestinal cancers and cancers of the prostate and the urinary tract predominantly occur at older ages.

In summary, there were some differences between estimated completeness and known completeness, but these deviations were limited, and their direction and magnitude was in agreement with knowledge on clinical aspects and clinical management of cancer patients, and with the circumstances of cancer registration in Saarland. We therefore conclude from our analyses that carefully conducted capture-recapture methods may be useful to delineate plausible ranges of completeness of cancer registration in the absence of independent sources against which completeness can be checked. Our analyses suggest, that performance of the capture-recapture method might be best among patients below age 75, for whom cancer registry data are typically more valid. Although their application for routine comparisons of completeness of registration between cancer registries may be limited to some extent by the availability of comparable sources of notification, capture-recapture estimates of completeness offer an alternative to traditional measures of completeness of population-based cancer registration, such as the 
DCO index or the mortality/incidence ratio. In contrast to these traditional measures, the capture-recapture estimates are direct estimates of completeness which can be used to compare completeness of cancers with strongly divergent case fatality rates.

Our analyses were restricted to the performance of two source capture-recapture methods within specific subgroups of patients to reflect the situation of many cancer registries which are based on two sources of notification only. More flexible strategies, such as use of loglinear models, can be used and less restrictive assumptions have to be made if more than two sources are available. ${ }^{4}$ For example, with three sources of ascertainment, all dependencies between pairs of sources can be taken into account by inclusion of appropriate two source interaction terms in a log-linear modelling approach. ${ }^{13}$ Nevertheless, the assumption of absence of three source interaction must still be made and this requires critical discussion. Furthermore, other problems, such as misclassification of cancer diagnoses on death certificates ${ }^{17}$ or less than perfect record linkage ${ }^{18}$ also require careful consideration. Further research should be devoted to the development of more refined capture-recapture methods that take these problems into account.

1 Coleman MP, Démaret E. Cancer registration in the European Community. Int $\mathcal{f}$ Cancer 1988;42:339-45.

2 Shanmugaratnam K. Availability and completeness of cancer registration worldwide. Rec Res Cancer Research 1989; 114:28-33.
3 Muir C, Waterhouse J, Mack T, Powell J, Whelan S, eds Cancer incidence in five continents. Vol V. Lyon: Internationa Agency for Research on Cancer, 1987. IARC Scientific Publication No 88.

4 Robles SC, Marrett LD, Clarke EA, Risch HA. An ap plication of capture-recapture methods to the estimatio of completeness of cancer registration. $\mathcal{f}$ Clin Epidemio 1988;41:495-501.

5 Cochi SL, Edmonds LE, Dyer K, et al. Congenital rubella syndrome in the United States, 1970-1985. On the verge of elimination. Am ₹ Epidemiol 1989;129:349-61.

6 LaPorte RE, McCarty DJ, Tull ES, Tajima N. Counting birds, bees and NCDs. Lancet 1992;339:494-5.

7 LaPorte RE, Tull ES, McCarty D. Monitoring the incidence of myocardial infarctions: applications of capture-mark recapture technology. Int $\mathcal{f}$ Epidemiol 1992;21:258-63.

8 Hilsenbeck SG, Kurucz C, Duncan RC. Estimation of completeness and adjustment of age-specific and agecompleteness and adjustment of age-specific and age-

9 McCarty DJ, Tull ES, Moy CS, Kwoh CK, LaPorte RE. McCarty DJ, Tull ES, Moy CS, Kwoh CK, LaPorte RE. Ascertainment corrected rates: applications of capt

10 Cormack RM. The statistics of capture-recapture methods. Oceanographic and Marine Biology. An Annual Review 1968 6:455-506.

11 Wittes J, Sidel VW. A generalization of the simple capturerecapture model with applications to epidemiological research. I Chron Dis 1968;21:287-301.

12 Wittes JT, Colton T. Capture-recapture methods for assessing the completeness of case ascertainment when usin multiple information sources. F Chron Dis 1974;27:25-36.

13 Bishop YMM, Fienberg SE, Holland PW. Discrete multivariate analysis: theory and practice. Cambridge, MA MIT Press, 1975:229-56.

14 Hook EB, Regal RR. Capture-recapture methods. Lancet 1992;339:742.

15 Hook EB, Regal RR. Validity of Bernoulli census, loglinear, and truncated binomial models for correcting for linear, and truncated binomial models for correcting for underestimates in

16 Parkin DM, Muir CS, Whelan SL, Gao Y-T, Ferlay J, Powell J. Cancer incidence in five continents. Vol VI. Lyon: International Agency for Research on Cancer, 1992. IARC Scientific Publication No 120

17 Percy C, Stanek E, Gloeckler L. Accuracy of cancer death certificates and its effect on cancer mortality statistics. $A m$ $\mathcal{f}$ Public Health 1981;71:242-50.

18 Storm $\mathrm{HH}$. Completeness of cancer registration in Denmark 1943-1966 and efficacy of record linkage procedures. In f Epidemiol 1988;17:44-9. 